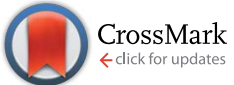

Cite this: RSC Adv., 2015, 5, 79423

Received 1st July 2015

Accepted 14th September 2015

DOI: $10.1039 / \mathrm{c} 5 \mathrm{ra12794g}$

www.rsc.org/advances

\section{Ceria nanoparticles with rhodamine $B$ as a powerful theranostic agent against intracellular oxidative stress $\uparrow$}

\author{
Nadezda Apostolova, +ł Susana Rovira-Llopis, $\dot{t}^{\mathrm{b}}$ Herme G. Baldoví, ${ }^{\mathrm{c}}$ Sergio Navalon, ${ }^{\mathrm{c}}$ \\ Abdullah M. Asiri, ${ }^{d}$ Victor M. Victor, ${ }^{b}$ Hermenegildo Garcia*cd \\ and Jose Raul Herance ${ }^{\star b e}$
}

Ceria nanoparticles with rhodamine B groups covalently attached on their surface (RhB-CeNPs) were successfully prepared to simultaneously exhibit antioxidant activity and the ability to detect oxidant species. In order to use them for biomedical purposes, the nanoparticles were internalized in two human cell lines (HeLa and Hep3B), confirmed by confocal microscopy. In addition, their biocompatibility was assessed by performing proliferation, viability and apoptosis assays, in which they showed a remarkable lack of toxicity. Thereafter, the antioxidant activity of RhB-CeNPs against reactive oxygen species (ROS) in a model of oxidative stress was demonstrated in HeLa cells using the dichloro-dihydro-fluorescein diacetate (DCFH-DA) assay. RhB-CeNPs exhibited higher cytosolic antioxidant activity than the wellestablished ceria nanoparticles. Surprisingly, the antioxidant capacity of RhB-CeNPs was evident when the ROS content of the cells increased notably (and was, therefore, harmful for those cells). Furthermore, the ability of RhB-CeNPs as ROS-content sensors was evaluated by measuring oxidative stress in HeLa cells using the intrinsic fluorescence of the rhodamine B groups present on the nanoparticles. The results with respect to the detection and quantification of ROS were similar to those obtained with DCFH-DA, a typical method of quantifying intracellular ROS. Our results demonstrate the potential of RhB-CeNPs as remarkably biocompatible theranostic agents with application in the field of oxidative stress.

\section{Introduction}

Oxidative stress is one of the main factors of cellular aging and other cellular disorders. ${ }^{\mathbf{1 - 4}}$ It is defined as an imbalance in the production of oxidant species and their effective removal by antioxidants and scavenger enzymes, and is one of the targets of therapies against diseases. In physiological conditions, a homeostatic balance exists between the formation of reactive

${ }^{a}$ Faculty of Health Sciences, Universitat Jaume I, Av. Vicent Sos Baynat s/n, 12071, Castellón de la Plana, Spain

${ }^{b}$ Service of Endocrinology, University Hospital Dr Peset, Foundation for the Promotion of Health and Biomedical Research in the Valencian Region (FISABIO) and CIBERehd, Av. Gaspar Aguilar 90, 46017, Valencia, Spain

${ }^{c}$ Instituto Universitario de Tecnología Química CSIC-UPV, Univ. Politécnica de Valencia, Av. De los Naranjos s/n, 46022, Valencia, Spain. E-mail: hgarcia@qim. upv.es; Fax: +34 963877809; Tel: +34 963877809

${ }^{d}$ Center of Excellence for Advanced Materials Research, King Abdulaziz University, Jeddah, Saudi Arabia

${ }^{e}$ Molecular Biology and Biochemistry Research Center for Nanomedicine, Vall d'Hebron Research Institute (VHIR), CIBBIM-Nanomedicine, CIBER-BBN, Passeig de la Vall d'Hebron 119-129, 08035 Barcelona, Spain. E-mail: raul.herance@vhir.org; Fax: +34 934894102; Tel: +34934893000 ext. 4668

$\dagger$ Electronic supplementary information (ESI) available: Additional scheme and figures. See DOI: 10.1039/c5ra12794g

\$ These authors have equally contributed to this work. oxygen species (ROS) and their elimination by endogenous antioxidant scavenging compounds and enzymes. ${ }^{5}$ The free radical hypothesis for tissue dysfunction postulates that ROS leads to a modification of lipids, proteins and nucleic acids, which, in turn, contributes to the etiology of different diseases. ${ }^{6}$ Importantly, low-to-moderate levels of ROS are physiologically normal and necessary for cell survival, while high levels are detrimental and can even cause cell death. For this reason, there is much current interest in developing new strategies to modulate ROS levels and, thus, control oxidative stress. ${ }^{7-11}$ With the aim of exploring in detail the role of ROS, several strategies have been tested in vivo and in vitro in order to evaluate the benefits of reducing oxidative stress in diseases such as cancer, diabetes, among others, with some beneficial effects being observed. The outcome of these studies has fuelled interest in developing new compounds and materials with antioxidant properties such as nanoparticles to control cellular oxidative stress.

Nanoparticles are used in different fields, including advanced materials, chemistry, biology and medicine, and their synthesis and properties have been topics of continuous study in recent decades. The structure of nanomaterials allows the possibility of incorporating several guests into their cavities when they are porous, or of covalently anchoring several 
functional components to their surface due to the high percentage of external atoms. Furthermore, the composition and chemical properties of nanoparticles can be designed to make them inert or reactive versus substrates or the environment. These features make nanoparticles particularly suitable for use in biology and biomedicine, where nanoparticles have been tested as drug delivery systems, and antioxidant or imaging agents, among other potential uses. ${ }^{12-17}$ In spite of the range of opportunities offered by nanoparticles, which can exhibit multifunctionality, the most common application in biomedicine only exploits a single property of each one. However, recently there has been increased interest in applying nanomaterials as bifunctional or multifunctional materials. The multifunctionality of nanoparticles derives from their complementary reactivity, which is due to the simultaneous incorporation of different functional groups and molecules in their structure and moreover the intrinsic properties of the modified nanoparticle. In this way, some nanomaterials have been designed to combine optical and magnetic resonance imaging, therapy and optical imaging, radiotherapy and imaging, magnetism and imaging, among other possibilities. ${ }^{18-22}$ In this context, one of the emerging applications of nanoparticles is theranosis, which combines therapy and imaging. In a related precedent, Zholobak et al. have recently described a type of nanoparticles that acts as theranostic agents for oxidative stress. $^{23}$ The reported system was composed by ceria nanoparticles with calcein molecules attached to the scaffold through ionic interaction, being sensitive for ROS related to oxidative stress. However, the system has the limitation that the dye may undergo decomposition by ROS and the measurements could underestimate the concentration of ROS by gradual loss of fluorescent emission. To avoid this limitation, we want to explore the possibility to obtain a ceria nanoparticle system with covalent attachment of a fluorophore that acts as fluorescent biosensor of the concentration of ROS in cells in which the fluorescence decrease as a function of ROS, and that can simultaneously act as antioxidant when the ROS concentration overcomes the damage threshold for cells. We believe it is possible to generate "intelligent" nanoparticulate materials in the field of theranostics to detect ROS content and to treat excess ROS, thus modulating antioxidant therapies in a harmless manner. This is the objective of the present work.

Ceria nanoparticles (CeNPs) have been found to be biocompatible with several cellular types, exhibiting antioxidant activity due to their redox properties, which are attributable to oxygen vacancies on their surface and the presence of $\mathrm{Ce}^{3+}$ ions in their structure. ${ }^{\mathbf{2 4 , 2 5}}$ In addition CeNPs are stable in aqueous media at neutral $\mathrm{pH}$ values. CeNPs have been described as free radical scavenger by several groups. ${ }^{26}$ The antioxidant activity of CeNPs can be enhanced by supporting metals in their structure, such as Au. ${ }^{27}$ The biocompatibility and redox properties of CeNPs have attracted interest from the field of biomedicine, and these nanoparticles have been proposed for some therapeutic applications. Accordingly, CeNPs have been tested in vitro and in vivo as quenchers of ROS in some types of cells, and as radiation protectors, cardioprotectors or reducers of endothelial inflammation, among other roles. ${ }^{28-32}$
Rhodamine B (RhB) is a fluorophore with typical absorption and fluorescence bands centered approximately at 550 and 580 $\mathrm{nm}$, respectively, and has been used for diverse purposes in chemistry, biology and medicine. The use of RhB derives mainly from its high fluorescence quantum yield and from the quenching of its fluorescence emission by energy transfer or electron transfer processes caused by the interaction of RhB with other compounds. ${ }^{33-37}$ In this sense, radical species or hydrogen peroxide $\left(\mathrm{H}_{2} \mathrm{O}_{2}\right)$ can oxidize $\mathrm{RhB}$, causing a decrease in fluorescence intensity that can correlate with the amount of oxidants, and is usually used to quantify the amount of oxidizing species. ${ }^{38-40}$ Thus, RhB could be used as a sensor of the oxidant species present in a sample. In addition, some rhodamine dyes are currently used for biomedical purposes such as probes for mitochondria and the endoplasmic reticulum, where it appears to be relatively non-toxic. ${ }^{\mathbf{4 1 - 4 3}}$

Due to the capacity of CeNPs as antioxidants and ROS scavengers, and the possibility of covalently attaching RhB to their surface to act as a sensor of oxidant species content, we have developed a novel theranostic nanoparticle to work both as a diagnostic agent of oxidant species content and a form of antioxidant therapy.

\section{Results and discussion}

In order to prepare a new class of nanomaterial with properties as both theranostic antioxidant and oxidant content sensor, RhB was covalently attached to CeNPs by modifying their surface. For this purpose, colloidal CeNPs were obtained by hydrolysis of $\mathrm{Ce}^{4+}$ salts in water at a controlled basic $\mathrm{pH}$ and purified by dialysis. The average nanoparticle size was determined from the width of the XRD peaks by applying the Scherrer equation, giving a value of $5.6 \mathrm{~nm}$ (Fig. S1, ESI $\dagger$ ). Additionally, the XRD pattern of nanoparticles confirmed that the sample corresponded with $\mathrm{CeO}_{2}$, as the XRD coincided with that reported for $\mathrm{CeO}_{2}$. Additionally, the UV-Vis spectrum of CeNPs was recorded. No bands at wavelengths longer than 400 nm were observed for CeNPs (Fig. 1). Once CeNPs had been

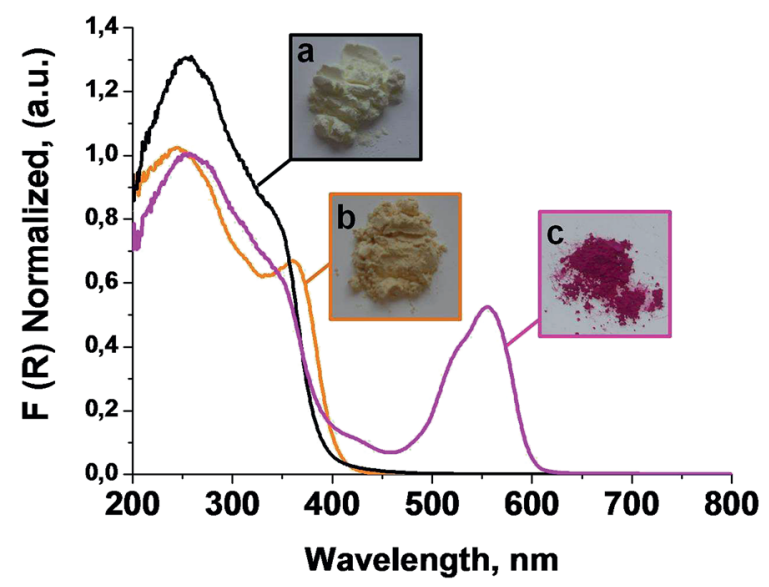

Fig. 1 Diffuse reflectance UV-Vis absorption spectra (plotted as the Kubelka-Munk function of the reflectivity, $R$ ) of CeNPs (a), $\mathrm{NH}_{2}-\mathrm{CeNPs}$ (b) and RhB-CeNPs (c) 


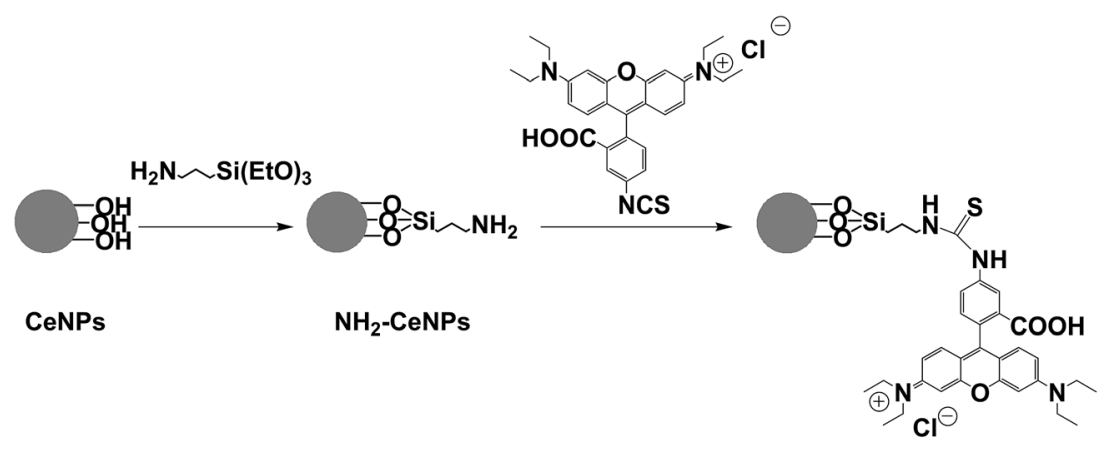

RhB-CeNPs

Scheme 1 Synthetic route followed to obtain $\mathrm{NH}_{2}-\mathrm{CeNPs}$ and RhB-CeNPs.

characterized, covalent functionalization of their surface with amino groups was carried out by silylation of the surface $\mathrm{OH}$ groups with 3-aminopropyltriethoxysilane in dry toluene. In this way, $\omega$-aminopropyl-functionalized CeNPs ( $\mathrm{NH}_{2}$-CeNPs) were obtained. This reaction takes place under neutral conditions and attaches the amino group through strong $\mathrm{Ce}-\mathrm{O}-\mathrm{Si}$ bonds. These amino groups act as tethers that connect RhB to CeNPs. Subsequent reaction of these aminated $\mathrm{NH}_{2}$-CeNPs with $\mathrm{RhB}$ isothiocyanate forms a strong thiourea linkage between the organic fluorophore and the inorganic CeNPs, as depicted in Scheme 1. The success of each synthesis step was assessed by chemical combustion analysis, FT-IR (Fig. 2) and diffuse reflectance UV-Vis spectroscopy (Fig. 1).

The presence of 3-aminopropyl groups anchored on the ceria surface was evident in the IR spectra of $\mathrm{NH}_{2}$-CeNPs; the two vibration bands characteristic of symmetric and asymmetric $\mathrm{NH}_{2}$ (3270 and $3230 \mathrm{~cm}^{-1}$ ) and $\mathrm{CH}_{2}$ stretching (2990 and 2950 $\mathrm{cm}^{-1}$ ) which were recorded as weak bands due to the low population of these groups. The UV-Vis spectrum of $\mathrm{NH}_{2}-\mathrm{CeNPS}$ shows a slight change in the color upon surface functionalization of CeNPs by $\omega$-aminopropyl groups. This spectral change could indicate the occurrence of some ligand to metal charge transfer complex between amino groups and of the silane and

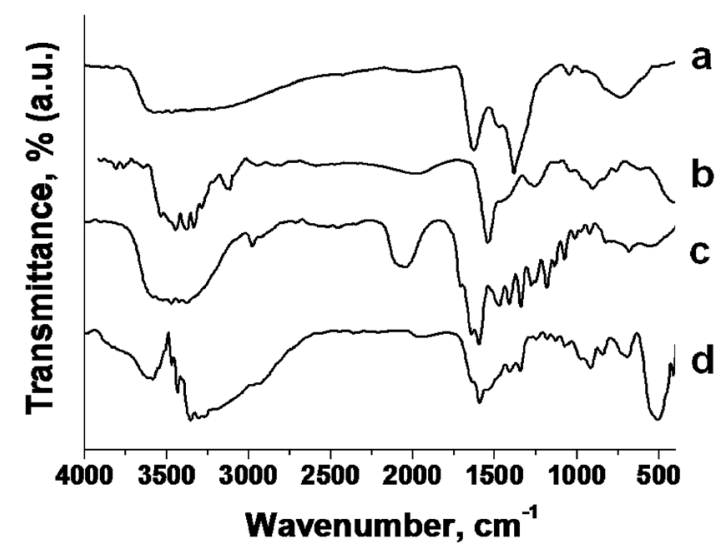

Fig. 2 FT-IR spectra of (a) CeNPs, (b) $\mathrm{NH}_{2}$-CeNPs, (c) rhodamine B isothiocyanate and (d) RhB-CeNPs.
$\mathrm{Ce}^{4+/ 3+}$ ions having free coordination positions. The loading of aminopropyl silyl groups on $\mathrm{NH}_{2}$-CeNPs was $4.1 \mathrm{mmol} \mathrm{g}^{-1}$ based on the $\mathrm{C}$ and $\mathrm{N}$ content in this material, which was determined by combustion chemical analysis. Additionally, the XRD pattern of $\mathrm{NH}_{2}$-CeNPs with respect to CeNPs shows that surface functionalization occurred without a significant increase in the average particle of CeNPs that remained at roughly $5.6 \mathrm{~nm}$ (Fig. S1, ESI $\dagger$ ). Subsequently, covalent attachment of $\mathrm{RhB}$ on $\mathrm{NH}_{2}$-CeNPs was carried out, producing a change in the colour of the $\mathrm{NH}_{2}$-CeNPs sample after functionalization that varied from light yellow to purple and was reflected in the diffuse reflectance spectra of the samples (Fig. 1). As shown in Fig. 1, the RhB-CeNPs sample exhibited an absorption band in the 500-600 $\mathrm{nm}$ region due to the anchored RhB dye. This RhB absorption band was somewhat broader than the optical spectrum of the rhodamine thioisocyanate in solution. This broadness is a general phenomenon observed when organic dyes are supported on solid surfaces and is due to the formation of aggregates and/or the inhomogeneity of the surface in terms of polarity and presence of $\mathrm{OH}$ groups. Furthermore, as expected for the presence of RhB in RhBCeNPs, this dye produced an intense emission. Fig. 3 shows the excitation spectrum showing that RhB-CeNPs emission is

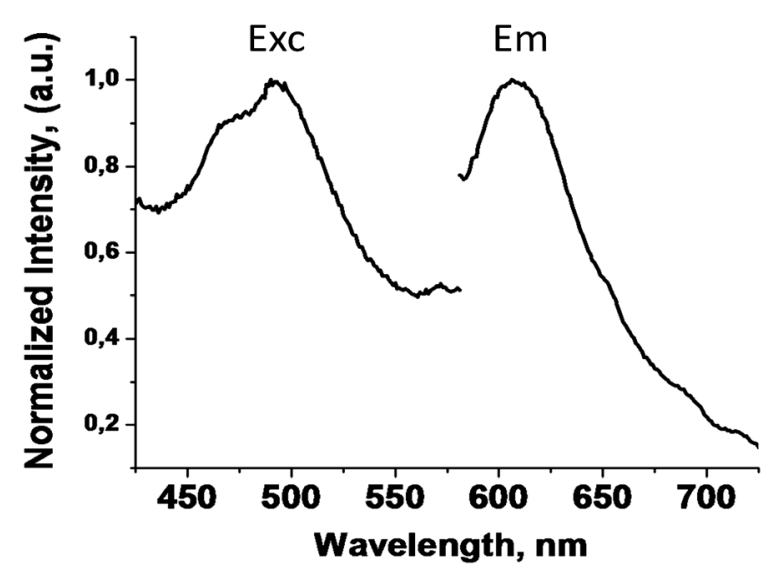

Fig. 3 Emission (Em) spectra recorded for RhB-CeNPs upon excitation at different excitation wavelengths $\left(\lambda_{\text {exc }} 425-575\right)$ showing that the emission spectrum does not depend on the excitation wavelength. 


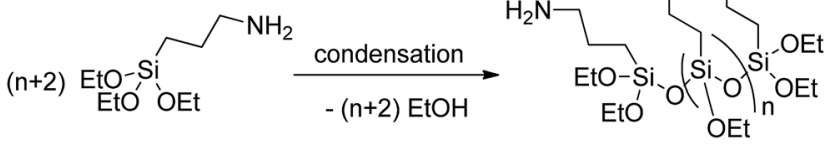

Scheme 2 Condensation of $\omega$-aminopropyl triethoxysilane forming oligomers and resulting in a high RhB loading on CeNPs.

observed upon excitation in a wide range of wavelengths going from 425 to $575 \mathrm{~nm}$, coinciding with the absorption band (see Fig. 1, plot c). No variation of the emission spectrum as a function of the excitation wavelength was observed and the emission maximum appeared at $600 \mathrm{~nm}$. A suitable excitation wavelength for maximum emission intensity was $510 \mathrm{~nm}$.

Additionally, the anchoring of RhB was confirmed by FT-IR spectroscopy, which provided firm evidence of the success of the incorporation. Thus, upon introduction of $\mathrm{RhB}$ isothiocyanate (Fig. 2, spectrum d) the isocyanate peak of the RhB isocyanate precursor appearing at $2055 \mathrm{~cm}^{-1}$ disappeared, but the characteristic narrow peaks corresponding with the aromatic region of the xanthene core were clearly resolved in the RhB-CeNPs spectrum (Fig. 2, spectrum d). Combustion chemical analysis of $\mathrm{S}$ allowed us to determine the loading of $\mathrm{RhB}$ in the resulting RhB-CeNPs, which was $3.2 \mathrm{mmol} \mathrm{g}^{-1}$, corresponding with about $80 \%$ of the $\mathrm{NH}_{2}$ groups present in RhBCeNPs. Considering that the specific surface area of $\mathrm{CeO}_{2}$ is $180 \mathrm{~m}^{2} \mathrm{~g}^{-1}$, these loading results in average about 100 molecules per $\mathrm{nm}^{2}$. This average is clearly much too high and indicates that Scheme 1 is an over simplification and there must be under the preparation conditions a certain degree of self condensation of $\omega$-aminopropyl triethoxysilanes as indicated in Scheme 2. These oligosiloxane condensates will be the species covalently attached to the surface of CeNPs.

To determine the particle size of RhB-CeNPs, a statistical analysis of RhB-CeNPs was performed by transmission electron microscopy (TEM), the size distribution revealing an average particle size of about $10 \mathrm{~nm}$ (Fig. 4).

In addition, RhB-CeNPs were submitted to dynamic light scattering study to determine their size distribution profile in suspension (Fig. S2, ESI $†$ ) and their zeta potential value (Fig. S3, ESI†). These data provide information about the surface

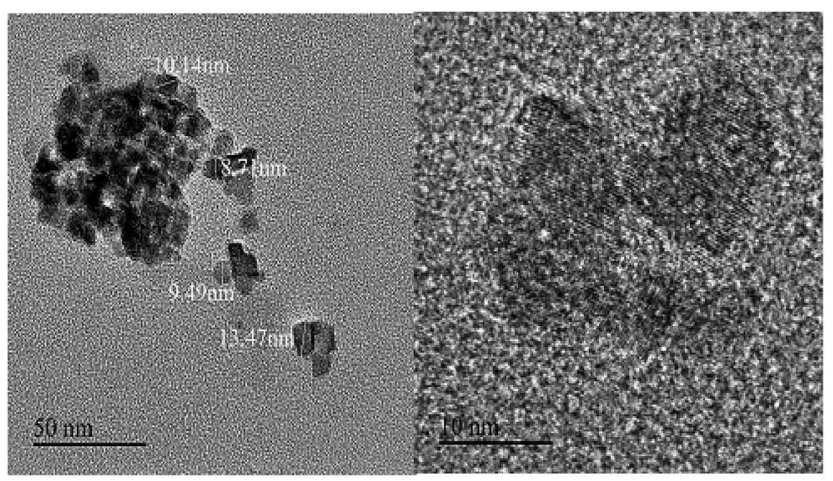

Fig. 4 Transmission electron microscopy recorded for RhB-CeNPs. modification of the CeNPs due to anchor RhB and the size and charge of the nanoparticles in aqueous solution, which is the medium used to biomedical purposes. Hydrodynamic size of RhB-CeNPs in water was about $54 \mathrm{~nm}$, which was higher than the value determined by TEM (Fig. 4), but this may have reflected partial agglomeration of the nanoparticles or, more likely, the presence of a cloud of water molecules solvating the RhB-CeNPs core. It should be noted that even though the surface of pristine nanoparticulate $\mathrm{CeO}_{2}$ is hydrophilic, upon modification with silanization and RhB dye the hydrophobicity of the particles has to increase. This hydrophobicity is responsible for the occurrence of some agglomeration in aqueous media to decrease the surface energy of the colloidal suspension. Regarding the stability of these nanoparticles, the zeta potential value at neutral $\mathrm{pH}$ was $-25 \mathrm{mV}$, which is within the limit to be considered a persistent colloid. This zeta potential value seems to be the balance between the intrinsic negative value of CeNPs and the positive charges introduced by RhB.

Once the RhB-CeNPs were obtained, their antioxidant capacity was assessed using the ABTS radical scavenger test. This test consists in measuring the decrease of absorbance corresponding to $\mathrm{ABTS}^{\circ+}$ (typically $410 \mathrm{~nm}$ ) when a radical scavenger or antioxidant is added to the solution. The test is carried out by following basically the methodology described by Seeram, et $a .^{46}$ Thus, initially $\mathrm{ABTS}^{\circ+}$ needs to be obtained by passing a colorless $5 \mathrm{mM}$ aqueous solution of ABTS through a filter containing $\mathrm{MnO}_{2}$. The resulting green solution of $\mathrm{ABTS}^{\cdot+}$ is centrifuged at $10000 \mathrm{rpm}$ for $10 \mathrm{~min}$ at $20^{\circ} \mathrm{C}$ to remove any trace of $\mathrm{MnO}_{2}$. Freshly prepared supernatant is used to assess the antioxidant capacity of RhB-CeNPs by monitoring the decrease of the corresponding $\mathrm{ABTS}^{+}$absorbance at $410 \mathrm{~nm}$ when different concentrations $\left(0,0.5,1,1.5\right.$, and $\left.2 \mathrm{mg} \mathrm{mL}^{-1}\right)$ of nanoparticles dispersed in PBS solution are added to the green solution. In order to avoid any possible interference in the UV$\mathrm{Vis} \mathrm{ABTS}^{++}$spectrum due to the addition of RhB-CeNPs, the UVVis spectrum of a RhB-CeNPs control solution is measured simultaneously to subtract to the radical absorbance spectrum. As shown in Fig. 5A, ABTS $^{++}$absorbance decreased gradually upon RhB-CeNPs addition. This fact confirms the antioxidant activity of the PBS solution of RhB-CeNPs and, therefore, the suitability of these nanoparticles as antioxidant therapeutic agents.

On the other hand, RhB-CeNPs have RhB groups covalent anchored to the CeNPs surface that could be used as sensors to quantify the content of oxidant species. This assumption is based on the well-known capacity of RhB to react with oxidants such as radical species and $\mathrm{H}_{2} \mathrm{O}_{2}$, among others. This quenching can be followed by the disappearance of the fluorescence band at $580 \mathrm{~nm}$, after excitation at $350 \mathrm{~nm}$, characteristic of the $\mathrm{RhB}$ groups present in RhB-CeNPs. These fluorescence experiments were performed with the same samples used to establish the antioxidant capacity of these nanoparticles (Fig. 5B), after checking for the absence of fluorescence originating from $\mathrm{ABTS}^{++}$or ABTS. Additionally, the capacity of RhB-CeNPs as oxidant content sensors was confirmed by a similar experiment in which the RhB-CeNPs content was maintained constant and the concentration of $\mathrm{ABTS}^{++}$was increased. For this purpose, 
A

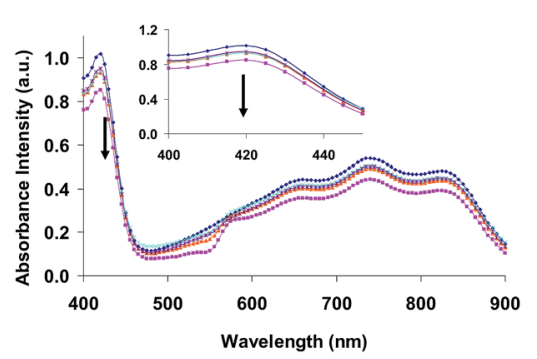

B

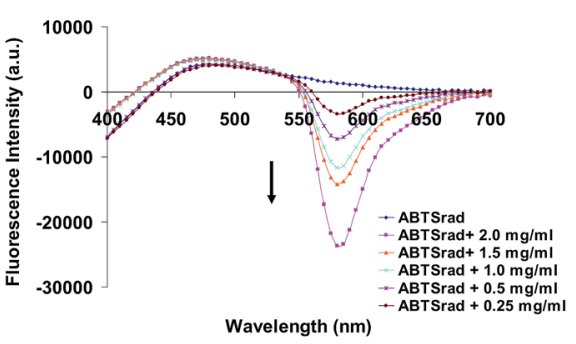

Fig. 5 UV-Vis (A) and fluorescent (b, $\lambda_{\text {ex }} 350 \mathrm{~nm}$ ), (B) spectra of ABTS radical cation after additioning different amounts of RhB-CeNPs. The insert shows the region where the decrease of the band is related with the radical scavenger or antioxidant assessment.

A

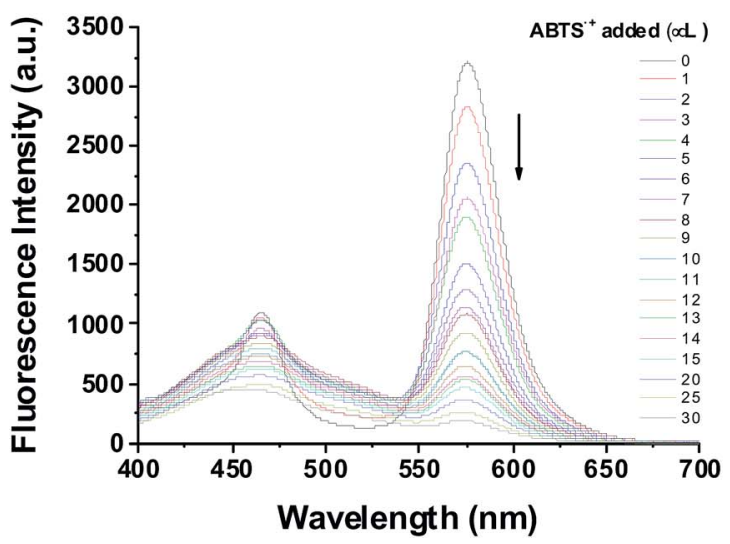

B

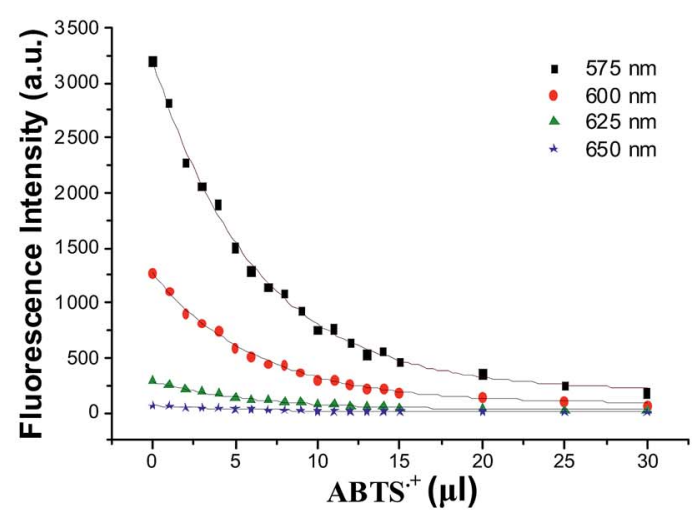

Fig. 6 (A) Fluorescent spectra of RhB-CeNPs in PBS solution recorded after the excitation at $350 \mathrm{~nm}$ and the addition of different aliquots of a 5 $\mathrm{mM} \mathrm{ABTS}^{\cdot+}$ aqueous solution. (B) Reduction of the fluorescence intensity of Rh-CeNPs solution at different wavelengths (575, 600, 625, 650 nm) after the addition of different aliquots of the ABTS ${ }^{++}$aqueous solution. All the fitting are successfully adjusted to a first order exponential plot $\left(575 \mathrm{~nm}: y=3007.815 \times \mathrm{e}^{(-x / 6.258)}+200.502, R^{2}=0.9965 ; 600 \mathrm{~nm}: y=1185720 \times \mathrm{e}^{(-x / 6.212)}+85.120, R^{2}=0.9972 ; 625 \mathrm{~nm}: y=260.635 \times\right.$ $\left.\mathrm{e}^{(-x / 6.351)}+22.877, R^{2}=0.9957 ; 650 \mathrm{~nm}: y=59.785 \times \mathrm{e}^{(-x / 6.281)}+5.668, R^{2}=0.9961\right)$.

the fluorescence spectra were recorded between 400-700 $\mathrm{nm}$ after exciting the sample at $350 \mathrm{~nm}$. In this experiment, the characteristic fluorescence intensity of RhB present on RhBCeNPs decreased progressively following the addition of ABTS $^{-+}$(Fig. 6A). The decrease of fluorescence intensity at different wavelengths (for example 575, 600, 625 and $650 \mathrm{~nm}$ ) followed a first order exponential plot versus the content of $\mathrm{ABTS}^{\cdot+}$ as an oxidant compound model (Fig. 6B), and confirmed the ability of RhB-CeNPs to quantify the amount of oxidant species. This capacity allows the determination of the concentration of ROS and $\mathrm{H}_{2} \mathrm{O}_{2}$, among others, in solution or in the cells, thus allowing their use as biosensors where the oxidant content may be observe by a decrease of the fluorescent band between 575-650 $\mathrm{nm}$ that correspond to RhB-CeNPs. In fact the antioxidant ability of RhB-CeNPs may be due to a combination of two processes coming from RhB and CeNPS because both have antioxidant capability. However, the initial antioxidant behavior is probably due mainly to $\mathrm{RhB}$ groups present on RhBCeNPs because the redox potential of $\mathrm{RhB}(-0.54 \mathrm{~V}$ approx $)$ is lower than CeNPs $(>1.00 \mathrm{~V}$, depending on the phase and size) ${ }^{44,45}$ In addition, the RhB emission band is very sensitive to $\mathrm{ABTS}^{\circ}+$ radicals as is observed at lower concentrations. Then,
CeNPs can act when the RhB groups are degraded or considerably diminished.

Before studying the antioxidant behaviour of RhB-CeNPs in cells, their ability to become internalized by cells was confirmed by confocal laser scanning microscopy, by which we monitored the characteristic red fluorescence of the $\mathrm{RhB}$ group present in the RhB-CeNPs. Fig. 7 shows a selected set of vertical $\mathrm{Z}$ confocal fluorescence images showing the intracellular location of RhBCeNPs nanoparticles (red spots). The cytoplasmic distribution of RhB-CeNPs can be observed clearly in the enlarged image of a single cell shown in Fig. 7 (lower panel). These results are compatible with previous reports that similar CeNPs samples can enter cells. ${ }^{15}$

Once RhB-CeNPs had been confirmed as both antioxidant agents and oxidant content sensors in aqueous solution and were shown to be internalized inside cells, their toxicity and capacity as sensors and antioxidants were evaluated in human cell culture models. Cytotoxicity is a major issue in the applicability of nanoparticles in biomedicine. For this reason, several experiments were performed to assess the effect of RhB-CeNPs on the viability, survival and proliferation of cultured cells. Two human cell lines (HeLa and Hep3B) were used in order to 
A
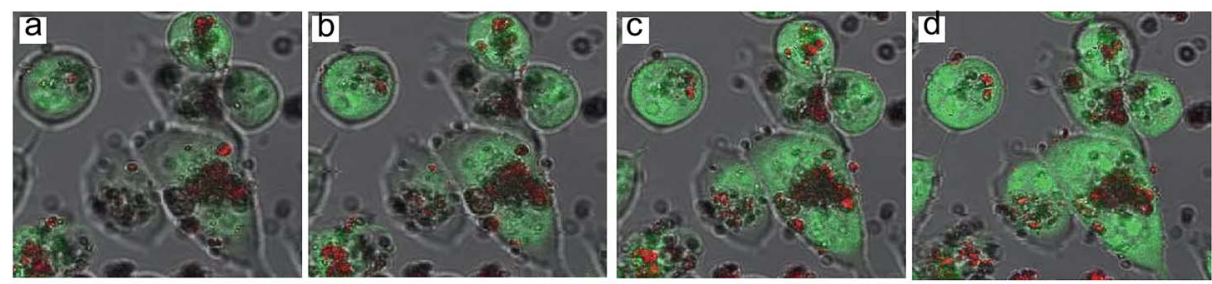

$\mathrm{B}$
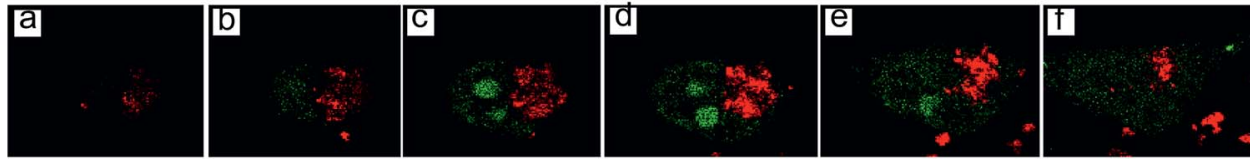

Fig. 7 Analysis of the cellular up-take of RhB-CeNPs by confocal microscopy. HeLa cells were exposed to the nanoparticles for $3 \mathrm{~h}$ and live imaging was performed thereafter. Representative images of $z$-stack (a to $d$ or a to f). (A) Images show the overlapping of light microscopy and fluorescence microcopy (the cytoplasm was stained with $\mathrm{N}$-acridine orange (NAO) fluorescing in green). (B) Single cell images show the fluorescent green signal coming from the nucleic acid stain SYTO80 whereas RhB-CeNPs autofluoresce in red.

avoid cell-type specificity. The results of the cell viability assessment (MTT assay) showed that the addition of CeNPs to the culture medium (incubation for $3 \mathrm{~h}$ followed by $21 \mathrm{~h}$ recovery) had no effect on the viability of either HeLa or Hep3B cells. RhB-CeNPs significantly diminished the viability of HeLa cells (approximately 50\%), whereas only a slight and nonsignificant decrease was detected in Hep3B cells (Fig. S4, ESI $\dagger$ ). Despite the susceptibility of the cellular viability depending on the cell line as well as nanoparticle concentration, RhB-CeNPs have similar viability data for Hep3B than the system described by Zholobak et al. ${ }^{23}$ Cell survival and exponential proliferation was studied further in both cell lines over a period of 3 days (fluorescence microscopy). RhB-CeNPs did not have a significant effect on cell survival or cell proliferation rate in either of the two cell lines, and results were similar to those observed for the control (untreated cells) at all the time points analyzed (24, 48 and 72 h). This suggests that the effect produced on cell viability in HeLa cells under treatment with RhB-CeNPs did not have major consequences on the survival of these fast proliferating cells over a longer period of time.

In order to confirm and expand the findings of the proliferation/survival experiments showing the lack of a cytotoxic effect, we determined the capacity of RhB-CeNPs to induce apoptosis in HeLa and Hep3B cells (incubation for $3 \mathrm{~h}$ followed by $21 \mathrm{~h}$ recovery). The widely used kinase inhibitor staurosporine was employed as a positive control for apoptotic induction. Several typical markers of apoptotic cell death were analyzed by fluorescence microscopy. An increase in mean Hoechst 44432 fluorescence (nuclear staining), indicative of increased chromatin condensation, which is a hallmark of apoptosis, was detected in both cell types after $24 \mathrm{~h}$-treatment with staurosporine. Of note, neither CeNPs nor RhB-CeNPs provoked any alteration in this parameter (Fig. S5A, ESI $†$ ). Similarly, a lack of apoptotic induction was observed when a Bivariate Annexin V/PI analysis was performed. Four distinct subpopulations of cells were evaluated: vital (Annexin $\mathrm{V}^{-} / \mathrm{PI}^{-}$), early apoptotic or classically apoptotic $\left(A n n e x i n \mathrm{~V}^{+} / \mathrm{PI}^{-}\right)$, late apoptotic/necrotic (Annexin $\mathrm{V}^{+} / \mathrm{PI}^{+}$), and damaged or classically necrotic (Annexin $\mathrm{V}^{-} / \mathrm{PI}^{+}$). The results are shown in Fig. S5B (see ESI†) (bar charts representing summary of the mean fluorescence data for both fluorochromes in both cell types). Fig. S5C (see ESI $\dagger$ ) depicts a representative cytogram of Hep3B cells in which the positive control (staurosporine $1 \mu \mathrm{M}$ ) triggered typical apoptosis in $45 \%$ of the cells compared to the presence of only $1.2 \%$ apoptotic cells in the negative control. CeNPs did not provoke a significant increase in the apoptotic subcellular population (3.77\% apoptotic cells in the presence of CeNPs). Moreover, there was a lack of a significant increase in the number of necrotic cells. This double staining experiment could not be performed for RhB-CeNPs due to the intense red fluorescence of RhB that interferes with the PI emission of the assay. To sum up, the obtained evidence indicates that shortterm exposure to the nanoparticles under study does not trigger cell death in cultured human cells.

The oxidant content of the cells was measured by means of the DCFH-DA assay, which is one of the most usual methods of detecting intracellular ROS for oxidative stress assessment. ${ }^{7,9,10}$ The results obtained when we tested the activity of CeNPs and RhB-CeNPs against oxidative stress in HeLa cells are shown in Fig. 8. Both CeNPs and RhB-CeNPs reduced the oxidant content of HeLa cells, but only at high rotenone concentrations $(125 \mu \mathrm{M}, P<0.05 ; 150 \mu \mathrm{M}, P<0.01)$, while the latter were more active in reducing cellular oxidative stress. There are numerous reports describing the anti-ROS activity of CeNPs, so it is expected that, when RhB are anchored to the surface of CeNPs, the antioxidant activity of these nanoparticles increases significantly, probably due to the tendency of the $\mathrm{RhB}$ group to undergo oxidation, as previously discussed. ${ }^{15,28,30}$ These data confirm the previous assumption that the antioxidant ability of RhB-CeNPs mainly comes from the RhB. In addition, despite using different cell lines, the antioxidant behavior of RhB-CeNPs against oxidative stress in cells is similar to the showed by Zholobak \& col using a 


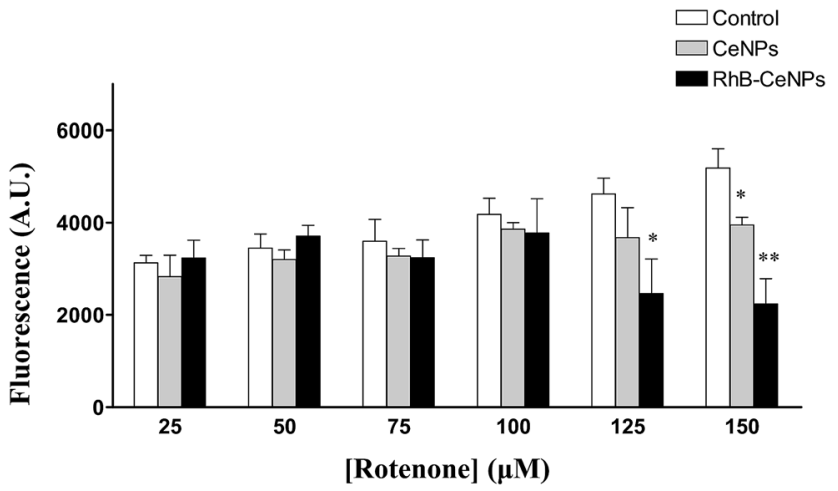

Fig. 8 Effect of CeNPs $\left(20 \mu \mathrm{g} \mathrm{mL}^{-1}\right)$, RhB-CeNPs $\left(20 \mu \mathrm{g} \mathrm{mL} \mathrm{m}^{-1}\right)$ on rotenone-induced ROS production. Bar charts showing DCFH fluorescence intensity in HeLa cells versus different rotenone concentration. Data (mean \pm SEM, $n=3$ ) were analyzed by 1 way ANOVA test, significance vs. control (untreated cells) $* p<0.05$ and $* * p<0.01$.

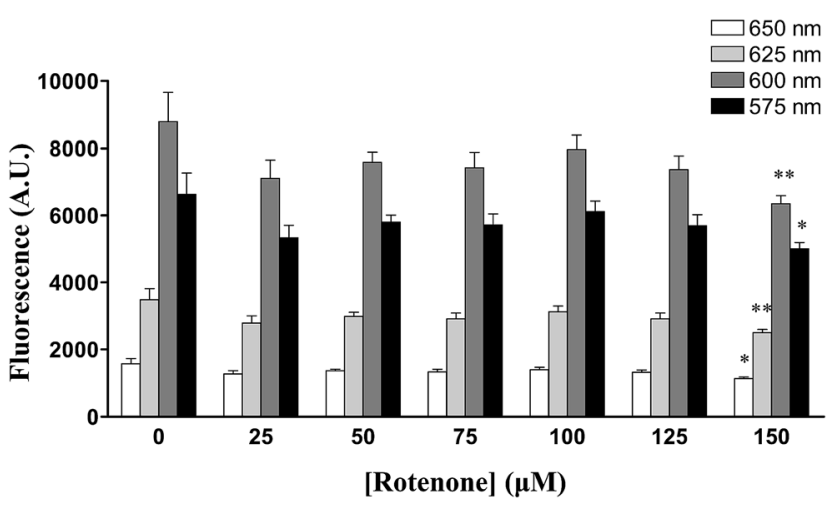

Fig. 9 Effect of applying different concentration of rotenone on the fluorescence intensity of RhB-CeNPs $\left(20 \mu \mathrm{g} \mathrm{mL}^{-1}\right)$ at different wavelengths $(575,600,625$ and $650 \mathrm{~nm}$ ) in HeLa cells. Data (mean $\pm \mathrm{SEM}, n$ $=8$ ) were analyzed by 1 way ANOVA test, significance vs. control (untreated cells) $* p<0.05$ and $* * p<0.01$.

calcein-CeNPs system where the scavenging of harmful ROS species is performed in presence of nanoparticles. ${ }^{23}$

On the other hand, the ability of RhB-CeNPs as ROS content sensors was studied by recording the fluorescence emission of cells which had been previously incubated with the same amount of RhB-CeNPs and after the addition of different rotenone amounts at 575, 600, 625 and $650 \mathrm{~nm}$. As shown in Fig. 9, the decrease in the fluorescence intensity at different wavelengths $(575,600,625$ and $650 \mathrm{~nm})$ owing to RhB-CeNPs was higher when the oxidative stress was increased by higher rotenone concentrations.

The data correlated with the results of the DCFH-DA probe assay, which is the most usual method of checking the ROS content of cells. These fluorescence intensity data confirm the capacity of RhB-CeNPs as a theranostic nanomaterial that acts simultaneously as a ROS quencher and biosensor. In addition, RhB-CeNPs as sensors have the additional advantage of being able to report at different wavelengths without losing sensor capacity for ROS species.

\section{Experimental section}

\section{Solvents and reagents}

Unless otherwise indicated, the solvents and reagents employed in both the preparation of materials and in cell culture were purchased from Sigma-Aldrich (Madrid, Spain), Scharlab (Sentmenat, Spain), Life Technologies (Madrid, Spain), or GIBCO (Invitrogen, Eugene, OR) and were used in the form in which they were supplied. The Annexin-V-FLUOS staining kit was from Roche Diagnostics (Sant Cugat del Vallés, Spain). Gases were supplied by Air-Liquide (Valencia, Spain). 96- and 48-well plates were purchased from Falcon BD (Madrid, Spain) and the Nunc 8well coverslip bottom chamber slides for confocal microscopy were supplied by Thermo Scientific (Madrid, Spain). 2,7Dichlorodihydrofluorescein diacetate (DCFH-DA) was purchased from Life Technologies (Madrid, Spain).

\section{Instrumentation}

Synthesis and characterization of nanoparticles. Combustion chemical analysis (C, H, N, and $\mathrm{S}$ ) were performed in a FISONS CHNOS analyzer. UV-Vis spectra were recorded in transmission mode on a Shimadzu UV-Vis spectrophotometer using quartz cuvettes of $1 \mathrm{~cm}$ path length. Fluorescence measurements were performed with a Synergy Mx plate reader fluorimeter (BioTek Instruments, Winooski, VT) or on a JASCO FP-8500 spectrofluorometer (Mary's Court, Easton, MD) provided with a monochromator of variable wavelength in the range of 200-900 nm. FT-IR spectra were recorded on a Nicolet 710 FT-IR spectrophotometer using $\mathrm{KBr}$ disks or self-supported wafers $(10 \mathrm{mg})$ compressed to 2 ton $\mathrm{cm}^{2}$ for $2 \mathrm{~min}$. Transmission electron microscopy (TEM) images were acquired by a Philips CM300 FEG system with an operating voltage of $100 \mathrm{kV}$ by placing microdrops of CeNPs suspensions directly onto a copper grid coated with carbon film (200 mesh). Powder XRD was recorded with a Philips X'Pert diffractometer using the $\mathrm{Cu} \mathrm{K}$ radiation at a scan rate of $2^{\circ} \mathrm{min}^{-1}$. Dynamic light scattering used to determine the size and zeta potential of colloidal aqueous solutions of nanoparticles was performed on a Zetasizer Nano ZS (Malvern Instrument, Malvern, UK). $5 \mathrm{mg}$ of nanoparticles were suspended in $100 \mathrm{~mL}$ for measurements, which were carried out in an automatic mode. The values of size and zeta potential are the average of 30 runs. The centrifuge used was a Hettich-Zentrifugen one (Tuttlingen, Germany).

Cell culture. The incubator used for cell culture was an IGO 150 (Jouan, Saint-Herblain, France). Confocal images were acquired using a Leica TCS-SP2 confocal laser scanning unit equipped with argon and helium-neon laser beams and attached to a Leica DM1RB inverted microscope. In the apoptosis studies, fluorescence was quantified using the IX81 Olympus fluorescence microscope coupled with static cytometry software "ScanR" version 2.03.2 Olympus (Hamburg, Germany) fluorescence measurements for ROS quantification were performed with a Synergy Mx plate reader fluorimeter (BioTek Instruments, Winooski, VT). Colorimetric analyses (MTT assay) were performed using a multiscan Thermo Labsystems platereader spectrophotometer (Thermoscientific, Rockford, IL). 


\section{Synthesis of rhodamine B-functionalized CeNPs (RhB-CeNPs)}

Synthesis of CeNPs. CeNPs were prepared by hydrolysis of $\mathrm{Ce}\left(\mathrm{NO}_{3}\right)_{4}$ at $\mathrm{pH}=9$ using $\left(\mathrm{NH}_{4}\right) \mathrm{OH}$ as reported. ${ }^{15}$ In brief, an ammonia aqueous solution $(1.12 \mathrm{~L}, 0.8 \mathrm{M})$ was added under constant stirring and at room temperature over $375 \mathrm{~mL}$ of $\mathrm{Ce}\left(\mathrm{NO}_{3}\right)_{4}(0.8 \mathrm{M})$, and the dispersion was heated in a poly(ethyleneterephthalate) vessel at $100{ }^{\circ} \mathrm{C}$ for $24 \mathrm{~h}$. The resulting yellow solid was filtered and dried under vacuum overnight. The success of the synthesis was determined by XRD, UV-Vis and FTIR. The surface area of CeNPs measured by isothermal $\mathrm{N}_{2}$ adsorption at $-198{ }^{\circ} \mathrm{C}$ using a Micromeritics ASAP2000 and applying the Brunauer-Emmett-Teller (BET) algorithm was 180 $\mathrm{m}^{2} \mathrm{~g}^{-1}$.

Preparation of 3-aminopropyl-functionalized CeNPs $\left(\mathrm{NH}_{2}-\right.$ CeNPs). Silylation of CeNPs was carried out by stirring a suspension of $1 \mathrm{~g}$ of dried CeNPs sample for $3 \mathrm{~h}$ under atmospheric pressure in $15 \mathrm{~mL}$ toluene containing $0.5 \mathrm{~mL}$ of 3 (aminopropyl)triethoxysilane at reflux temperature for $2 \mathrm{~h}$. Afterwards, the mixture was allowed to cool at room temperature and $\mathrm{NH}_{2}$-CeNPs was decanted and washed with fresh toluene aliquots $(3 \times 2 \mathrm{~mL})$ and allowed to dry, again at room temperature. The percentage of aminopropyl groups was determined by combustion elemental analysis and the success of the functionalization was further assessed by UV-Vis, XRD and FT-IR.

Preparation of rhodamine B-functionalized CeNPs (RhBCeNPS). $0.5 \mathrm{~g}$ of $\mathrm{NH}_{2}$-CeNPs was suspended in $15 \mathrm{~mL}$ of $\mathrm{CH}_{2} \mathrm{Cl}_{2}$, to which $0.100 \mathrm{~g}$ of rhodamine $\mathrm{B}$ thioisocyanate was added. The suspension was stirred magnetically at reflux temperature for 4 h. After this time, the solid was collected and washed with fresh $\mathrm{CH}_{2} \mathrm{Cl}_{2}$ until it became colorless $(7 \times 20 \mathrm{~mL})$. The resulting RhB-CeNPs was dried at room temperature under reduced pressure. Following this, nanoparticles were characterized by combustion elemental analysis, TEM, FT-IR, dynamic light scattering and UV-Vis/fluorescence spectroscopy.

\section{Assessment of RhB-CeNPs as antioxidant and radical sensor agents}

2,2-Azino-bis-3-ethylbenzthiazoline-6-sulfonic acid (ABTS) radical cation solution for radical scavenger-antioxidant assays. ABTS radical cation $\left(\mathrm{ABTS}^{\cdot+}\right.$ ) was prepared following the methodology described by Seeram, et al. with minor changes. ${ }^{46}$ In short, a $5 \mathrm{mM}$ water solution of ABTS was passed through a Whatman filter containing $\mathrm{MnO}_{2}$. The resulting green solution was centrifuged at $5000 \mathrm{rpm}$ for $10 \mathrm{~min}$ at $20{ }^{\circ} \mathrm{C}$ and the supernatant solution was used immediately for radical scavenger-antioxidant experiments.

Assessment of the antioxidant behaviour of RhB-CeNPs. We prepared $50 \mu \mathrm{l}$ of freshly prepared $\mathrm{ABTS}^{\cdot+}$ solution and $200 \mu \mathrm{L}$ of several RhB-CeNPs solutions at different concentrations $(0,0.5$, $1,1.5$, and $2 \mathrm{mg} \mathrm{mL} \mathrm{m}^{-1}$ ) in phosphate buffered saline solution (PBS). These mixtures were poured into a 96-well plate and stirred for $10 \mathrm{~min}$. After this time, absorption and fluorescence spectra $\left(\lambda_{\text {exc }} 350 \mathrm{~nm} / \lambda_{\text {em }} 400-700 \mathrm{~nm}\right)$ were recorded. This experiment was performed in triplicate using three replicates in each case.
Assessment of RhB-CeNPs as antioxidant sensors. Aliquots of $1 \mu \mathrm{L}$ of freshly prepared ABTS $^{++}$solution were added consecutively to a $0.1 \mathrm{mg} \mathrm{mL}^{-1}$ solution of RhB-CeNPs in PBS, until a total of $15 \mu \mathrm{L}$, and, from that point, aliquots of $5 \mu \mathrm{L}$ were added up until a total of $30 \mu \mathrm{L}$. Each mixture was stirred in the dark at room temperature for $30 \mathrm{~min}$ before measuring fluorescence, which was done after sample excitation at $350 \mathrm{~nm}$. Fluorescence was recorded between 400-700 nm.

\section{Cell culture}

Experiments were performed with the human hepatoma Hep3B (ATCC HB-8064) and the human cervical carcinoma HeLa (ATCC CCL-2) cell lines. Reagents employed in cell culture were purchased from GIBCO. Hep3B cells were cultured in minimum essential medium (MEM) supplemented with $1 \mathrm{mM}$ nonessential amino acids, whereas HeLa cells were cultured in Dulbecco's modified Eagle's medium (DMEM) with a high glucose concentration $\left(4.5 \mathrm{mg} \mathrm{mL}^{-1}\right)$. Both culture media were supplemented with $10 \%$ heat-inactivated fetal bovine serum (FBS) and the antibiotics penicillin $50 \mathrm{U} \mathrm{mL}^{-1}$ and streptomycin $50 \mu \mathrm{g}$ $\mathrm{mL}^{-1}$. Cells were maintained in an incubator at $37{ }^{\circ} \mathrm{C}$ in a humidified atmosphere of $5 \% \mathrm{CO}_{2} / 95 \%$ air.

Confocal laser scanning microscopy images. HeLa cells were seeded at 10000 cells per well in Nunc 8-well coverslip bottom chamber slides. Treatment with RhB-CeNPs was performed over $3 \mathrm{~h}$, after which cells were incubated for a further $30 \mathrm{~min}$ with 5 $\mu \mathrm{M}$ Syto80, a green fluorescent marker which preferentially stains nucleic acids, or $2.5 \mu \mathrm{M} 10 \mathrm{~N}$ nonyl-acridine orange (NAO) in order to stain the cytoplasm. Subsequently, the cell culture was washed twice with Hank's Balanced Salt Solution (HBSS) and immediately submitted to image acquisition using confocal microscopy. The excitation wavelengths for the fluorochromes were $488 \mathrm{~nm}$ for Syto 80 and $543 \mathrm{~nm}$ for RhB-CeNPs, and the emission apertures for fluorescence detection were 510-540 $\mathrm{nm}$ for Syto80 and 575-650 $\mathrm{nm}$ for RhB-CeNPs. The Zsection series were obtained by progressive increments of $1.0 \pm$ $0.2 \mu \mathrm{m}$.

Proliferation and viability. Cells were seeded in 48-well plates one day before the experiment at 15000 and 5000 cells per well for Hep3B and HeLa, respectively. Incubation in the presence of RhB-CeNPs (at $20 \mu \mathrm{g} \mathrm{mL}^{-1}$ ) took place for $3 \mathrm{~h}$, after which the medium was refreshed and cells were allowed to proliferate exponentially for 3 days. Cell count was performed at 24,48 , and $72 \mathrm{~h}$ by means of fluorescence microscopy coupled with static cytometry. For this, $1 \mu \mathrm{M}$ of the chromatin-staining dye Hoechst 33342 was added for the last 30 min of treatment and the cells were washed with HBSS. All treatments were performed in duplicate, and 25 images per well were recorded with a IX81 Olympus fluorescence microscope coupled with static cytometry software "ScanR" version 2.03.2.

In addition, the 3-[4,5-dimethylthiazol-2-yl]-2,5-diprenyl tetrazolium bromide (MTT) assay was performed. MTT is a colorimetric assay based on the ability of cells to reduce a soluble yellow tetrazolium salt to blue formazan crystals. ${ }^{9}$ This reduction takes place only when mitochondrial reductase enzymes are active and is, thus, a marker of cell viability related 
to mitochondrial function. For these experiments, cells were seeded in 96-well plates at 20000 and 50000 cells per well for Hep3B and HeLa, respectively. Incubation with RhB-CeNPs (at $20 \mu \mathrm{g} \mathrm{mL}^{-1}$ ) was performed over $3 \mathrm{~h}$, after which the medium was refreshed and cells were further cultured for a total of a 24 h. MTT reagent (Roche Diagnostics, Mannheim, Germany) was added ( $20 \mu \mathrm{L}$ per well) for the last $4 \mathrm{~h}$ of the treatment. Cells were solubilized with dimethyl sulfoxide (DMSO) $(100 \mu \mathrm{L}$ per well, $5 \mathrm{~min}, 37^{\circ} \mathrm{C}$ ), and absorbance was detected using a Thermo Labsystems Multiscan plate-reader spectrophotometer. Results were obtained by subtracting the intensity of the signal at $690 \mathrm{~nm}$ from the $570 \mathrm{~nm}$ absorbance, as indicated in the instructions provided with the kit.

Apoptosis. Induction of apoptosis was studied by Bivariate Annexin V/PI analysis for which HeLa and Hep3B cells were seeded in duplicate in 48-well plates at 20000 and 30000 cells per well respectively. Staurosporine (STS), a widely used protein kinase inhibitor, was employed as a positive proapoptotic control using $0.1 \mu \mathrm{M}$ and $1 \mu \mathrm{M}$ for HeLa and Hep3B, respectively. ${ }^{9}$ Incubation with RhB-CeNPs (at $20 \mu \mathrm{g} \mathrm{mL}{ }^{-1}$ ) was performed over $3 \mathrm{~h}$, after which the medium was refreshed and cells were cultured for a further period of $24 \mathrm{~h}$. The medium was then replaced with HBSS containing $1 \mu \mathrm{M}$ of Hoechst 33342 to stain nuclei and $0.9 \mu \mathrm{L}$ Annexin V-fluorescein in order to detect phosphatidylserine exteriorization, and cells were incubated in the dark for $20 \mathrm{~min}$ at $37^{\circ} \mathrm{C}$. Thereafter, $0.3 \mu \mathrm{L}$ of the chromatin detecting dye propidium iodide (PI) was added ( $5 \mathrm{~min}$ ) to label dead or damaged cells. Annexin V and PI solutions were purchased in the form of the Annexin-V-FLUOS staining kit. Analysis was performed of 25 images per well recorded with a IX81 Olympus fluorescence microscope coupled with static cytometry software "ScanR" version 2.03.2.

\section{ROS production and assessment of RhB-CeNPs as antioxidant agents and sensors}

To assess ROS content, the day before the experiment HeLa cells were seeded in 96-well plates at 20000 cells per well in Dulbecco's Modified Eagle's Medium (DMEM) containing 10\% heat inactivated fetal bovine serum, $2 \mathrm{mM}$ L-glutamine, $1 \mathrm{mM}$ sodium pyruvate, $50 \mu \mathrm{g} \mathrm{mL}^{-1}$ penicillin and $50 \mu \mathrm{g} \mathrm{mL}^{-1}$ streptomycin.

Culture medium was removed from cells and fresh medium with or without $20 \mu \mathrm{g} \mathrm{mL}{ }^{-1}$ RhB-CeNPs and CeNPs were added. Cells were incubated for $3 \mathrm{~h}$ at $37^{\circ} \mathrm{C}$ under gentle agitation. The medium was then replaced with preheated PBS containing 2.5 $\mu \mathrm{M}$ 2,7-dichlorodihydrofluorescein diacetate (DCFH-DA). After incubation for $30 \mathrm{~min}$ at $37^{\circ} \mathrm{C}$ in the dark under gentle agitation, various concentrations of rotenone, a widely used pharmacological inductor of ROS generation were added and fluorescence was measured at $5 \mathrm{~min}$ intervals over a $30 \mathrm{~min}$ period in a Synergy $\mathrm{Mx}$ plate reader (BioTek Instruments). DCFH-DA fluorescence intensity was recorded after $30 \mathrm{~min}$ of rotenone treatment at $535 \mathrm{~nm}$ and after exciting the sample at $485 \mathrm{~nm}$. Fluorescence intensity in each rotenone condition was calculated by subtracting the fluorescence emitted by untreated cells.
To assess the ability of RhB-CeNPs as ROS biosensors we performed the same experiment as described previously, but without the addition of DCFH-DA. Fluorescence intensity of $\mathrm{RhB}$ was also measured at 5 min-intervals during a $30 \mathrm{~min}$ period after exciting the sample at $543 \mathrm{~nm}$ and recording the emission at 575, 600, 625, and $650 \mathrm{~nm}$. The RhB fluorescence values shown are raw data after $30 \mathrm{~min}$ of rotenone treatment.

\section{Conclusion}

In the present study, ceria nanoparticles with rhodamine B groups covalently attached to their surface have been successfully prepared and characterized. These RhB-CeNPs exhibit antioxidant activity and sensor capacity against oxidant species both in solution and human cells submitted to oxidative stress. In addition, we have confirmed that RhB-CeNPs can cross the cellular membrane and are remarkably biocompatible without increasing cell mortality. In these cells, RhB-CeNPs present a higher antioxidant capacity than the well-established CeNPs when there are harmful levels of ROS content, and exhibit a similar sensor capacity to the DCFH-DA probe. Thus, we present RhB-CeNPs as a new class of nanomaterial with theranostic properties for oxidative stress.

\section{Acknowledgements}

The present work was supported by the grant CP13/00252, PI13/ 1025 from Carlos III Health Institute, and by the European Regional Development Fund (ERDF). In addition, this study was financed by the Spanish Ministry of Economy and Competitiveness (Severo Ochoa and CTQ2012-32315), the Generalitat Valenciana (Prometeo 2012-013), Foundation for the Promotion of Health and Biomedical Research in the Valencian Region (UGP-14-095) and supported by the Spanish Ministry of Science and Innovation.

\section{Notes and references}

1 C. Espinet, H. Gonzalo, C. Fleitas, M. J. Menal and J. Egea, Curr. Drug Targets, 2015, 16, 20.

2 M. Matsuo, J. Clin. Biochem. Nutr., 2004, 35, 63.

3 R. S. Sohal and R. Weindruch, Science, 1996, 273, 59.

4 G. Vitale, S. Salvioli and C. Franceschi, Nat. Rev. Endocrinol., 2013, 9, 228.

5 M. Rocha, N. Apostolova, J. R. Herance, S. Rovira-Llopis, A. Hernandez-Mijares and V. M. Victor, Med. Res. Rev., 2014, 34, 160.

6 J. M. Gutteridge and J. Mitchell, Br. Med. Bull., 1999, 55, 49.

7 C. Gorrini, I. S. Harris and T. W. Mak, Nat. Rev. Drug Discovery, 2013, 12, 931.

8 C. Gutierrez-Merino, C. Lopez-Sanchez, R. Lagoa, A. K. Samhan-Arias, C. Bueno and V. Garcia-Martinez, Curr. Med. Chem., 2011, 18, 1195.

9 R. Martín, C. Menchón, N. Apostolova, V. M. Victor, M. Alvaro, J. R. Herance and H. García, ACS Nano, 2010, 4, 6957. 
10 L. Raj, T. Ide, A. U. Gurkar, M. Foley, M. Schenone, X. Li, N. J. Tolliday, T. R. Golub, S. A. Carr, A. F. Shamji, A. M. Stern, A. Mandinova, S. L. Schreiber and S. W. Lee, Nature, 2011, 475, 231.

11 L. Rochette, M. Zeller, Y. Cottin and C. Vergely, Biochim. Biophys. Acta, 2014, 1840, 2709.

12 B. Y. Kim, J. T. Rutka and W. C. Chan, N. Engl. J. Med., 2010, 363, 2434.

13 S. E. Lohse and C. J. Murphy, J. Am. Chem. Soc., 2012, 134, 15607.

14 A. H. Lu, E. L. Salabas and F. Schueth, Angew. Chem., Int. Ed., 2007, 46, 1222.

15 C. Menchón, R. Martín, N. Apostolova, V. M. Victor, M. Alvaro, J. R. Herance and H. García, Small, 2012, 8, 1895.

16 T. K. Sau, A. L. Rogach, F. Jaeckel, T. A. Klar and J. Feldmann, Adv. Mater., 2010, 22, 1805.

17 V. Valtchev and L. Tosheva, Chem. Rev., 2013, 113, 6734.

18 J. Della Rocca, D. Liu and W. Lin, Acc. Chem. Res., 2011, 44, 957.

19 D. E. Lee, H. Koo, I. C. Sun, J. H. Ryu, K. Kim and I. C. Kwon, Chem. Soc. Rev., 2012, 41, 2656.

20 J. Liu, X. Zheng, L. Yan, L. Zhou, G. Tian, W. Yin, L. Wang, Y. Liu, Z. Hu, Z. Gu, C. Chen and Y. Zhao, ACS Nano, 2015, 9, 696.

21 K. Riehemann, S. W. Schneider, T. A. Luger, B. Godin, M. Ferrari and H. Fuchs, Angew. Chem., Int. Ed., 2009, 48, 872.

22 V. Wagner, A. Dullaart, A. K. Bock and A. Zweck, Nat. Biotechnol., 2006, 24, 1211.

23 N. M. Zholobak, A. B. Shcherbakov, E. O. Vitukova, A. V. Yegorova, Y. V. Scripinets, I. I. Leonenko, A. Y. Baranchikov, V. P. Antonovich and V. K. Ivanov, RSC Adv., 2014, 4, 51703.

24 F. Esch, S. Fabris, L. Zhou, T. Montini, C. Africh, P. Fornasiero, G. Comelli and R. Rosei, Science, 2005, 309, 752.

25 S. Turner, S. Lazar, B. Freitag, R. Egoavil, J. Verbeeck, S. Put, Y. Strauven and G. van Tendeloo, Nanoscale, 2011, 3, 3385.

26 J. T. Dahle and Y. Arai, Int. J. Environ. Res. Public Health, 2015, 12, 1253.

27 A. Maldotti, A. Molinari, R. Juárez and H. Garcia, Chem. Sci., 2011, 2, 1831.
28 A. Y. Estevez, S. Pritchard, K. Harper, J. W. Aston, A. Lynch, J. J. Lucky, J. S. Ludington, P. Chatani, W. P. Mosenthal, J. C. Leiter, S. Andreescu and J. S. Erlichman, Free Radical Biol. Med., 2011, 51, 1155.

29 A. Gojova, J. T. Lee, H. S. Jung, B. Guo, A. I. Barakat and I. M. Kennedy, Inhalation Toxicol., 2009, 21, 123.

30 C. Korsvik, S. Patil, S. Seal and W. T. Self, Chem. Commun., 2007, 10, 1056.

31 J. Niu, A. Azfer, L. M. Rogers, X. Wang and P. E. Kolattukudy, Cardiovasc. Res., 2007, 73, 549.

32 R. W. Tarnuzzer, J. Colon, S. Patil and S. Seal, Nano Lett., 2005, 5, 2573.

33 S. S. Banerjee and D. H. Chen, Nanotechnology, 2009, 20, 185103.

34 M. Das, D. Mishra, P. Dhak, S. Gupta, T. K. Maiti, A. Basak and P. Pramanik, Small, 2009, 5, 2883.

35 D. Shi, M. Ni, J. Luo, M. Akashi, X. Liu and M. Chen, Analyst, 2015, 140, 1306.

36 E. Vlashi, L. Kelderhouse, J. E. Sturgis and P. S. Low, ACS Nano, 2013, 7, 8573.

37 L. Yuan, W. Lin, K. Zheng, L. He and W. Huang, Chem. Soc. Rev., 2013, 42, 622.

38 A. Mehrdad and R. Hashemzadeh, Ultrason. Sonochem., 2010, 17, 168.

39 P. Qu, J. Zhao, T. Shen and H. Hidaka, J. Mol. Catal. A: Chem., 1998, 129, 257.

40 X. Zhou, J. Lan, G. Liu, K. Deng, Y. Yang, G. Nie, J. Yu and L. Zhi, Angew. Chem., Int. Ed., 2012, 51, 178.

41 J. H. Kwak, Y. He, B. Yoon, S. Koo, Z. Yang, E. J. Kang, B. H. Lee, S. Y. Han, Y. C. Yoo, K. B. Lee and J. S. Kim, Chem. Commun., 2014, 50, 13045.

42 A. Reisch, P. Didier, L. Richert, S. Oncul, Y. Arntz, Y. Mély and A. S. Klymchenko, Nat. Commun., 2014, 5, 4089.

43 P. Reungpatthanaphong, S. Dechsupa, J. Meesungnoen, C. Loetchutinat and S. Mankhetkorn, J. Biochem. Biophys. Methods, 2003, 57, 1.

44 G. V. Zakharova, V. E. Korobov, V. V. Shabalov and A. K. Chibisov, J. Appl. Spectrosc., 1983, 39, 765.

45 V. Amstutz, K. E. Toghill, F. Powlesland, H. Vrubel, C. Comninellis, X. Hu and H. H. Girault, Energy Environ. Sci., 2014, 7, 2350.

46 N. P. Seeram, S. M. Henning, Y. Niu, R. Lee, H. S. Scheuller and D. Heber, J. Agric. Food Chem., 2006, 54, 1599. 